\title{
The Causative Organism of Urinary Tract Infections UTI: a Cross-sectional Study from a Tertiary Hospital in Saudi Arabia
}

\author{
Mohammed Alwaladali, Maya Soufan, and Bandar S. \\ Almutairi
}

\begin{abstract}
BACKGROUND: Urinary tract infections (UTIs) are a common disease with a high burden on the healthcare industry. A systematic exploration is necessary of the organisms that cause UTIs, to improve empirical management of patients with acute illness before culture results are obtained. The prevalence of these organisms, both in immunocompetent and immunocompromised patients in Saudi Arabia, needs further clarification.
\end{abstract}

METHODS: This is a cross-sectional analysis reviewing the charts of patients visiting the emergency department of, and those admitted to, a tertiary hospital in Saudi Arabia during the month of JulyAugust 2021.

RESULT: The total number of participants was 199, with a mean age of 55.8 years $(S D=20.02)$, ranging from 14 to 97 years. $61.8 \%$ of patients were immunocompromised, and one-third were from the oncology wards. $40.7 \%(n=81)$ were diabetics and $8.54 \%(n=17)$ had CKD/ESRD. Females were 25\% more likely than males to acquire Candida infections, while Pseudomonas aeruginosa was $26.7 \%$ more prevalent in males. Nevertheless, mixed gramnegative bacteria caused the most UTIs, in $40.20 \%$ $(n=80)$ of cases, followed by Candida, $16.1 \%(n=32)$, and then mixed gram-positive bacteria, in $14.57 \%$ $(n=29)$. This was also observed among immunocompromised patients and the subsegment of oncology patients on active chemotherapy, although with variable percentages.

CONCLUSION: This study demonstrates that patients' immune status is the main determinant of the causative organism of UTIs. The treatment threshold

Mohammed Alwaladali is with Emergency Medicine, King Fahd Medical City, e-mail: mohammed.667@hotmail.com

Maya Soufan is with Alfaisal University, e-mail: maya.soufan@gmail.com

Bandar S. Almutairi is with Presidency of State Security, email:Drbssm@gmail.com for Candida in diabetic patients and those with CKD/ESRD should be lower, especially when they require admission.

Index Terms-Urinary tract infection, Urine Culture

\section{INTRODUCTION}

Urinary tract infections (UTIs) are a common disease, accounting for $20-25 \%$ of all infections and representing 150 million diagnosed cases annually [1-3]. The incidence of such infections differs in relation to age and gender. In adult women, for instance, the lifetime incidence of UTI is greater than $50 \%$ [2], while urosepsis is the second-most common cause of sepsis among the elderly population $[4,5]$. It has been estimated that UTIs cost approximately $\$ 800,000$ annually in a local study [6]. As such, the burden of the disease and its relatively high prevalence mandate revisiting this infection and exploring it in greater depth.

There are several predispositions to the development of UTI beyond the anatomical and physiological factors, including sexual activity, immunosuppression, and the use of Foley catheters. Therapeutic use of Foley catheters, for instance, increases the risk for UTI by $10 \%$ for every day the catheter is in situ [7,8]. Meanwhile, immunosuppression promotes the development of UTI due to the loss of the body's defense system [9]. This is seen among people with diabetes, chronic kidney disease, organ transplants, and patients on active chemotherapy. Those with diabetes appear to be affected more than the others. In Saudi Arabia, for example, the prevalence of UTI is around one quarter in those with diabetes mellitus [10], making it the most prevalent infection in this cohort $[11,12]$. 
Furthermore, the organisms that cause UTI vary depending on the patients' comorbidities, previous infections, the use of Foley catheters and instrumentation, and prior surgeries. Classically, Escherichia coli is the most common organism, followed by Klebsiella pneumoniae $[13,14]$. The former remains the most common irrespective of whether the patient is immunocompromised or immunocompetent [9]. In addition, other organisms, including Enterococcus species, Candida, Staphylococcus aureus, Proteus mirabilis, Pseudomonas aeruginosa, and group B Streptococcus (GBS), were also cultured [13].

The prevalence of these organisms in immunocompetent and immunocompromised patients in Saudi Arabia needs further elucidation. Furthermore, an updated exploration of the topic is necessary, primarily for physicians working under acute conditions who treat patients empirically before obtaining culture results. We therefore aim, in this study, to explore the organisms causing UTI in emergency patients and inpatients of a tertiary hospital in Saudi Arabia. In addition, we aim to determine whether the type of organism causing the UTI can be predicted on the basis of the patient's demographic or immune status.

\section{METHODS}

This is a cross-sectional analysis reviewing the charts of patients visiting the emergency department and those admitted during the month of July-August 2021. We tracked patients from the E.D., inpatients in the internal medicine and oncology wards, and those in the intensive care unit. Our sample was selected from a large tertiary hospital in the capital of Saudi Arabia, with a capacity of 1,200 beds.

We estimated a sample size of 200 patients using Raosoft@ online sample size calculator (95\% Confidence interval and $6.33 \%$ margin of error). Using only information from the medical records, we obtained the demographic details, urine culture method, and whether a clean catch or via a Foley catheter. We also noted any comorbidities, and whether the patient was known to have cancer and was on chemotherapy. Patients were labelled as immunocompromised if they had diabetes, chronic kidney disease/end-stage renal disease, or were on active chemotherapy.
In our analysis, we used the mean and the standard deviation to report the patients' age, and used proportions to register other demographic details. We used Fisher's exact test to explore the presence of a nonrandom association between the organisms causing UTI and the patients' demographics or immune status. We used STATA ${ }^{\circledR}$ to analyse the results, with a $\mathrm{p}<0.05$ threshold for statistically significant findings. The IRB board approves this study, with IRB registration number 00010471

\section{RESULTS}

\section{Demographics:}

The total number of participants was 199. The mean age of the patients was 55.8 years $(\mathrm{SD}=20.02)$, ranging from 14 to 97 years. The patients' demographic details are illustrated in Table $1.61 .8 \%$ of the patients were immunocompromised, and onethird were from the oncology wards. $40.7 \%(n=81)$ were diabetic, and $8.54 \%(\mathrm{n}=17)$ had chronic kidney disease or end-stage renal disease. Most of the urine samples were obtained via clean catch, with $12 \%$ via a Foley catheter.

\section{Organisms causing UTI:}

Most UTIs were caused by mixed gram-negative bacteria, as seen in $40.20 \%(n=80)$ of the sample, followed by Candida, in $16.1 \% \quad(n=32)$, and then mixed gram-positive bacteria, in $14.57 \%(n=29)$. Escherichia coli was cultured in $7.04 \%(n=14)$ of the patients. Other organisms are illustrated in Table 2.

In oncology patients, the most common organisms causing UTI were mixed gram-negative bacteria, in $45.8 \%(n=22)$ of patients, followed by Candida and mixed gram-positive bacteria, both seen in $12.5 \%$ $(n=6)$. The proportions of the various organisms in oncology patients are illustrated in Table 3.

Similarly, the most common organisms among immunocompromised patients were mixed gramnegative bacteria, representing $42.3 \%(n=52)$ of total infections in this patient cohort. Candida caused $21.1 \%(n=26)$ of UTI in this stratum, followed by mixed gram-positive bacteria, at $12.2 \%(n=15)$. Table 4 illustrates the prevalence of these organisms according to a subgroup analysis of immunocompromised and immunocompetent patients. 
TABLE I

PARTICIPANTS' DEMOGRAPHICS

\begin{tabular}{ll} 
Demographics & Proportion \% (n) \\
Gender & \\
Male & $43.72(87)$ \\
Female & $56.28(112)$ \\
Oncology patients & $24.12(48)$ \\
Diabetics & $40.7(81)$ \\
CKD/ESRD & $8.54(17)$ \\
Source of urine sample & \\
Clean catch & $80.90(161)$ \\
Foley catheter & $12.06(24)$ \\
Health status & \\
Immunocompetent & $36.68(73)$ \\
Immunocompromised & $61.81(123)$ \\
\hline
\end{tabular}

CKD: Chronic kidney disease; ESRD: End-stage renal disease

TABLE II

ORGANISMS CAUSING UTI IN OUR SAMPLE

\begin{tabular}{ll}
\hline Organism(s) & Proportion \% (n) \\
Candida & $16.08(32)$ \\
Enterobacter cloacae & $1.01(2)$ \\
Enterobacter faecalis & $1.01(2)$ \\
Escherichia coli & $7.04(14)$ \\
Klebsiella pneumoniae & $4.02(8)$ \\
Mixed gram-negative bacteria & $40.20(80)$ \\
Mixed gram-negative and positive bacteria & $7.54(15)$ \\
Mixed gram-negative bacteria and yeast & $3.02(6)$ \\
Mixed gram-positive bacteria & $14.57(29)$ \\
Proteus mirabilis & $2.51(5)$ \\
Pseudomonas aeruginosa & $3.02(6)$ \\
\hline
\end{tabular}

TABLE III

ORGANISMS CAUSING UTI IN ONCOLOGY PATIENTS

\begin{tabular}{|c|c|c|}
\hline Organism(s) & Oncology patient $\%$ (n) & Non-oncology patients \% (n) \\
\hline Candida & $12.5(6)$ & $17.6(26)$ \\
\hline Enterobacter cloacae & 0 & $1.4(2)$ \\
\hline Enterobacter faecalis & $4.2(2)$ & 0 \\
\hline Escherichia coli & $8.3(4)$ & $6.8(10)$ \\
\hline Klebsiella pneumonia & $8.3(4)$ & $2.7(4)$ \\
\hline Mixed gram-negative bacteria & $45.8(22)$ & $37.8(56)$ \\
\hline Mixed gram-negative and positive bacteria & $6.3(3)$ & $8.1(12)$ \\
\hline Mixed gram-negative bacteria and yeast & 0 & $4.1(6)$ \\
\hline Mixed gram-positive bacteria & $12.5(6)$ & $14.8(22)$ \\
\hline Proteus mirabilis & 0 & $3.4(5)$ \\
\hline Pseudomonas aeruginosa & $2.1(1)$ & $3.4(5)$ \\
\hline Total & $100(48)$ & $100(148)$ \\
\hline
\end{tabular}


TABLE IV

ORGANISMS CAUSING UTI IN IMMUNOCOMPROMISED AND IMMUNOCOMPETENT PATIENTS

\begin{tabular}{lll}
\hline Organism(s) & Immunocompromised \% (n) & Immunocompetent \% (n) \\
Candida and other yeast & $21.1(26)$ & $8.2(6)$ \\
Enterobacter cloacae & 0 & $2.7(2)$ \\
Enterobacter faecalis & $1.6(2)$ & 0 \\
Escherichia coli & $7.3(9)$ & $6.8(5)$ \\
Klebsiella pneumonia & $4.1(5)$ & $4.1(3)$ \\
Mixed gram-negative bacteria & $42.3(52)$ & $35.6(26)$ \\
Mixed gram-negative and positive bacteria & $7.3(9)$ & $8.2(6)$ \\
Mixed gram-negative bacteria and yeast & $1.6(2)$ & $5.5(4)$ \\
Mixed gram-positive bacteria & $12.2(15)$ & $17.8(13)$ \\
Proteus mirabilis & $0.8(1)$ & $5.5(4)$ \\
Pseudomonas aeruginosa & $1.6(2)$ & $5.5(4)$ \\
Total & $100(123)$ & $100(73)$ \\
\hline
\end{tabular}

Age and urine organisms:

Non-parametric analysis was conducted to explore the association between age and the organism cultured from the urine. We were unable to uncover a significant association; $\mathrm{p}=0.07$.

\section{Gender and urine organisms:}

We used Fisher's exact test to determine the association between patients' gender and the cultured organism, $p=0.049$. We noted that females were more likely to acquire Candida infection than males, with a difference of $25 \%$. Pseudomonas aeruginosa was $26.7 \%$ more in males. Furthermore, females were more likely to acquire Escherichia coli and Klebsiella, with a difference of $25.7 \%$ and $25 \%$ respectively. Enterobacter faecalis was only seen in females. Mixed gram-negative bacteria were more in males, with a $25 \%$ difference.

\section{Immune status and urine organisms:}

The patients' immune status also revealed an association with the cultured organisms using Fisher's exact test. Immunocompromised patients were more likely than their immunocompetent counterparts to have Candida, with a difference of $51.25 \%$. Mixed gram-negative bacteria were also common among the former segment, seen in 50.7\%. Enterobacter faecalis was only cultured from immunocompromised patients, whereas Enterobacter cloacae was only seen in the immunocompetent; $\mathrm{p}=0.031$.

Fisher's exact test was also used to analyse the difference between organisms causing UTI in oncology patients versus those seen in non-oncology patients. Candida was more common in the latter, with a $67.7 \%$ difference. Moreover, Enterobacter cloacae was only cultured from non-oncology patients, while none of this cohort had Enterobacter faecalis. However, the difference was not statistically significant, $\mathrm{p}=0.23$.

\section{Foley catheters and urine organisms:}

Urine samples obtained using Foley catheters did not differ significantly from clean catch samples with regard to the organism implicated; $X^{2}=9.93$, $p=0.54$.

\section{DISCUSSION}

It is crucial to explore the prevalence of the causative organisms for UTIs within the different geographic regions. In our analysis, mixed gramnegative bacteria were far more common than Escherichia coli. Indeed, this emerges as the most common cause among patients admitted with UTI, in general and for those with diabetes, CKD/ESRD, and oncology patients on active treatment. These findings are distinct and incongruent with other studies in which E. coli was the most prevalent uropathogen, followed by Klebsiella and Staphylococcus [15-18].

Candida was observed among diabetic and CKD/ESRD patients, and less among oncology patients. Our sample revealed a total of 32 patients with Candida in their urine culture, representing $16.08 \%$ of all UTIs in this sample. Only six patients were oncology patients, representing $12.5 \%$, whereas the prevalence of Candida increased to $21.1 \% \quad(n=26)$ when we included diabetic and CKD/ESRD patients. It is also important to note 
Candida's prevalence among immunocompetent patients in our sample was $8.2 \%(n=6)$, indicating that there is still a high infection burden among the immunocompetent. Nevertheless, immunocompromised patients are prone to invasive candidiasis [19], which should be considered when managing critically ill patients.

Mixed gram-positive bacteria were seen more among immunocompetent and non-oncology patients. Whether this bacterium needs to be covered empirically with antibiotics in the E.D. requires further analysis.

It is prudent to assert that our study examined patients in the E.D., those admitted to various wards, and in the ICU. Therefore, patients discharged beyond the E.D. might not be represented appropriately. This might suggest a sampling bias, as more severe UTI is represented in our sample than in those treated and discharged. Further analysis of discharged patients is needed.

\section{CONCLUSION}

This study demonstrates that patients' immune status is the main determinant of the causative organism of UTIs. As such, the treatment threshold for Candida in diabetic patients and those with CKD/ESRD should be lower, especially when they require admission. Emergency physicians should provide antibiotics that cover gram-negative bacteria for patients in general, Candida for immunocompromised patients, and gram-positive bacteria for immunocompetent patients.

\section{REFERENCES}

[1] Foxman B. Epidemiology of urinary tract infections: incidence, morbidity, and economic costs. Am J Med. 2002 Jul 8;113 Suppl 1A:5S-13S. doi: 10.1016/s0002-9343(02)01054-9. PMID: 12113866.

[2] Medina M, Castillo-Pino E. An introduction to the epidemiology and burden of urinary tract infections. Ther Adv Urol. 2019 May 2;11:1756287219832172. doi: 10.1177/1756287219832172. PMID: 31105774 ; PMCID: PMC6502976.

[3] Cunha MA, Assunção GL, Medeiros IM, Freitas MR. Antibiotic Resistance Patterns Of Urinary Tract Infections In A Northeastern Brazilian Capital. Rev Inst Med Trop Sao Paulo. 2016;58:2. doi: 10.1590/S1678-9946201658002. Epub 2016 Feb 23.
[4] Martín S, Pérez A, Aldecoa C. Sepsis and Immunosenescence in the Elderly Patient: A Review. Front Med (Lausanne). 2017 Feb 28;4:20. doi: 10.3389/fmed.2017.00020. PMID: 28293557; PMCID: PMC5329014.

[5] Mayr FB, Yende S, Angus DC. Epidemiology of severe sepsis. Virulence. 2014 Jan 1;5(1):4-11. doi: 10.4161/viru.27372. Epub 2013 Dec 11. PMID: 24335434 ; PMCID: PMC3916382.

[6] Alanazi MQ. An evaluation of communityacquired urinary tract infection and appropriateness of treatment in an emergency department in Saudi Arabia. Ther Clin Risk Manag. 2018;14:2363-2373. doi: $10.2147 /$ tcrm.S178855

[7] Lo E, Nicolle LE, Coffin SE, Gould C, Maragakis LL, Meddings J, Pegues DA, Pettis AM, Saint S, Yokoe DS. Strategies to prevent catheter-associated urinary tract infections in acute care hospitals: 2014 update. Infect Control Hosp Epidemiol. 2014 May;35(5):464-79. doi: 10.1086/675718. PMID: 24709715.

[8] Sabih A, Leslie SW. Complicated Urinary Tract Infections. [Updated 2021 Aug 12]. In: StatPearls [Internet]. Treasure Island (F.L.): StatPearls Publishing; 2021 Jan-. Available from: https://www .ncbi.nlm.nih.gov/books/NBK436013/

[9] Tandogdu Z, Cai T, Koves B, Wagenlehner F, Bjerklund-Johansen TE. Urinary Tract Infections in Immunocompromised Patients with Diabetes, Chronic Kidney Disease, and Kidney Transplant. Eur Urol Focus. 2016 Oct;2(4):394-399. doi: 10.1016/j.euf.2016.08.006. Epub 2016 Oct 5. PMID: 28723471.

[10] Al-Rubeaan KA, Moharram O, Al-Naqeb D, Hassan A, Rafiullah MR. Prevalence of urinary tract infection and risk factors among Saudi patients with diabetes. World J Urol. 2013 Jun;31(3):573-8. doi: 10.1007/s00345-012-0934-x. Epub 2012 Sep 7. PMID: 22956119.

[11] Bonadio M, Boldirini E, Forrotti G, Matteucci E, Vigna A, Mori S, Giampietro O (2004). Asymptomatic bacteriuria in women with diabetes: influence of metabolic control. Clin Infect Dis 38: e41-e45 .

[12] Boyco EJ, Fihn SD, Scholes D, Abraham L, Monsey B (2005). Risk of urinary tract infection and asymptomatic bacteriuria among diabetic and nondiabetic postmenopausal women. Am J Epidemiol 
161:557-564.

[13] Flores-Mireles AL, Walker JN, Caparon M, Hultgren SJ. Urinary tract infections: epidemiology, mechanisms of infection and treatment options. Nat Rev Microbiol. 2015 May;13(5):269-84. doi: 10.1038/nrmicro3432. Epub 2015 Apr 8. PMID: 25853778 ; PMCID: PMC4457377.

[14] Stamm WE. An epidemic of urinary tract infections? N Engl J Med. 2001 Oct 4;345(14):10557. doi: 10.1056/NEJM200110043451409. PMID: 11586959 .

[15] Sime WT, Biazin H, Zeleke TA, Desalegn Z. Urinary tract infection in cancer patients and antimicrobial susceptibility of isolates in Tikur Anbessa Specialized Hospital, Addis Ababa, Ethiopia. PLoS One. 2020 Dec 10;15(12):e0243474. doi: 10.1371/journal.pone.0243474. PMID: 33301465; PMCID: PMC7728278.

[16] Parikh P, Bhat V. Urinary tract infection in cancer patients in a tertiary cancer setting in India: microbial spectrum and antibiotic susceptibility pattern. Antimicrob Resist Infect Control. 2015 Jun 16;4(Suppl 1):P221. doi: 10.1186/2047-2994-4-S1P221. PMCID: PMC4475055.

[17] Shrestha G, Wei X, Hann K, Soe K.T, Satyanarayana S, Siwakoti B, Bastakoti S, Mulmi R, Rana K, Lamichhane N. Bacterial Profile and Antibiotic Resistance among Cancer Patients with Urinary Tract Infection in a National Tertiary Cancer Hospital of Nepal. Trop. Med. Infect. Dis. 2021, 6, 49. https://doi.org/ 10.3390/tropicalmed6020049

[18] Abiye Tigabu, Worku Ferede, Gizeaddis Belay, Baye Gelaw. Prevalence of Asymptomatic Bacteriuria and Antibiotic Susceptibility Patterns of Bacterial Isolates among Cancer Patients and Healthy Blood Donors at the University of Gondar Specialized Hospital. International Journal of Microbiology, vol. 2020, Article ID 3091564, 9 pages , 2020. https://doi.org/10.1155/2020/3091564

[19] Delaloye J, Calandra T. Invasive candidiasis as a cause of sepsis in the critically ill patient. Virulence. 2014 Jan 1;5(1):161-9. doi: 10.4161/viru.26187. Epub 2013 Aug 27. PMID: 24157707; PMCID: PMC3916370. 